\title{
Interpersonal Synchronization Protects Against the Antisocial Outcomes of Frustration
}

Psychological Reports

2022, Vol. 0(0) I-24

(C) The Author(s) 2022

Article reuse guidelines:

sagepub.com/journals-permissions

DOI: |0.|I77/0033294|2| I05477|

journals.sagepub.com/home/prx

@SAGE

\section{Karol Dybowski ',*, Barbara Raczka',*, Svetlana Postarnak', São Luís Castro', and Susana Silva' \\ 'Center for Psychology at University of Porto, Faculty of Psychology and Education Sciences, Porto, Portugal}

\begin{abstract}
Prosociality improves with interpersonal synchronization-the temporal coordination of movement across individuals. We tested whether the benefits of interpersonal synchronization extend to temporary circumstances of induced frustration, where negative changes in prosociality are expected as a result. Participants performed two joint tasks-synchronization versus non-synchronization. Each task was performed twice, with high versus low induced frustration. After each joint task, prosociality was measured both with explicit tests, in which participants were aware of the test goal, and implicit ones, where they were less aware. Frustration levels per task were also reported. Results showed that increase in frustration led to decrease in implicit prosociality after the non-synchronization task, but not after synchronization, suggesting that interpersonal synchronization attenuates the antisocial outcomes of frustration. In addition, our study highlights the advantages of implicit measures of prosociality, among which the test we created (Interpersonal Trust Test) may stand as a useful resource in future experimental research.
\end{abstract}

*Joint first authors.

\section{Corresponding Author:}

Susana Silva, Center for Psychology at University of Porto, Faculty of Psychology and Education Sciences, Rua Alfredo Allen, s/n, Porto 4200-I35, Portugal.

Email: susanamsilva@fpce.up.pt 


\section{Keywords}

Prosocial behavior, interpersonal synchronization, frustration, new questionnaires, socially desirable responding

\section{Introduction}

Prosocial behavior is a multifaceted construct (Padilla-Walker \& Carlo, 2015) that refers to voluntary actions intended to benefit someone other than oneself (Brief \& Motowidlo, 1986), including actions such as help, comforting, sharing, and cooperation (Batson \& Powell, 2003). Altruistic behaviors are one particular and extreme aspect of prosociality (tendency for prosocial behaviors), in that altruism engages the willingness to make personal sacrifices for the benefit of others (Ellis et al., 2018) without any expectation of material or social reward (Walster \& Piliavin, 1972). Interpersonal trust is often viewed as a proxy of prosociality since both are intimately related: beliefs in other's prosociality (trust) improve one's own prosociality (Ahmed \& Salas, 2013); trust predicts prosocial behaviors (Christian Cadenhead \& Richman, 1996; Rotenberg et al., 2005); both trust and prosocial behaviors result from group cohesion (Bos et al., 2018); and lack of interpersonal trust blocks the prosocial tendency of intuitive decisions (Rand \& Kraft-Todd, 2014). Some authors consider trust as part of prosociality (e.g., Keltner et al., 2014). In the present study, we look at altruism and interpersonal trust as two facets of prosociality.

Prosociality is often viewed as a trait (e.g., Skoe et al., 2002) rather than a state, suggesting that an individual's level of prosociality tends to be stable across situations. A recent meta-analysis showed that situational factors such as mood or the presence of bystanders are far from accounting for helping behaviors (Lefevor et al., 2017). Some research results have even shown that unlike Machiavellianism, prosociality seems impervious to situational influences (Bereczkei et al., 2010). This dominant trait-like view of prosociality makes sense considering the well-known role of typically longlasting influences such as genetics, culture, and family environment (parenting styles and parental models) on prosocial behaviors (Ando \& Kawamoto, 2021; Knafo \& Israel, 2010; Knafo \& Plomin, 2006; Knafo-Noam et al., 2015; Luria et al., 2015). However, the debate has not been closed yet, and the possibility that prosociality may change according to circumstances seems to be real.

Challenging the apparent irrelevance of situational factors, recent research findings raised the hypothesis that the circumstance of having one's movements overlapping in time with other people's movements - interpersonal synchronization (Bernieri et al., 1988; Foubert et al., 2017) could foster prosociality (see Keller et al., 2014, for a review). Musical contexts would be particularly effective, in that joint movement would be facilitated by a shared musical beat (Stupacher et al., 2017). Interpersonal synchronization promotes the willingness to help (Cirelli et al., 2014; Hu et al., 2017; Reddish et al., 2013) and has positive effects on interpersonal cooperation (Anshel \& Kipper, 1988; Fairhurst et al., 2013; Kaufman, 2004; Kirschner \& Tomasello, 2010; 
Reddish et al., 2013; Wiltermuth \& Heath, 2009) as well as on trust (Anshel \& Kipper, 1988; Launay et al., 2013). The link between interpersonal synchronization and prosociality seems deeply related to social bonding mechanisms: joint movement coordination facilitates the attentional union between individuals as well as their ability to anticipate their partner's actions (Keller et al., 2014; Koban et al., 2019). Such an increased perception of the other person elicits increased affiliation (Cacioppo et al., 2014; Hove \& Risen, 2009), feelings of connectedness (Marsh et al., 2009; Rennung \& Göritz, 2016) and empathy (Behrends et al., 2012), which may in turn be responsible for fostering prosocial behavior. From an evolutionary viewpoint, it is likely that groups who favored coordinated (synchronized) action coupled with internal dispositions for help, cooperation and trust (prosociality) may have excelled in survival due to increased combat power (Reddish et al., 2013; Roederer, 1984) or general synergy. While coordinated gestures may provide the basis for the group's survival, they may only allow so when motor coordination coexists with a prosocial direction (Reddish et al., 2013). In this view, interpersonal synchronization may only make sense when it goes along with prosociality.

The idea that interpersonal synchronization increases prosociality implies that the reverse is true, that is, that interpersonal synchronization decreases antisocial behaviors such as non-cooperation or aggression. One particularity of antisocial behaviors and attitudes is that they are often reactive, in that they may respond to transient states of frustration (Blair, 2001; Giacalone \& Greenberg, 1997; Miller, 1941). While previous studies have addressed the ways in which interpersonal synchronization may change participants' basal levels of prosociality, they have not, to our knowledge, examined whether it changes the transient, reactive states of antisociality that tend to follow frustration. Thus, one question remains open: could it be that the known effects of interpersonal synchronization on prosociality are able to counteract the transient antisociality that typically emerges from frustration states? An affirmative answer would be consistent with evidence from non-humans: in pigs, the presence of a congener with which social bonds had been established plays a protective role against the behavioral consequences of frustration (Arnone \& Dantzer, 1980). Given that interpersonal synchronization seems to be a matter of social bonding (see above), it is reasonable to expect that it has the same type of protective role. From a practical viewpoint, the possibility of using interpersonal synchronization as a tool for intervening in transient antisocial states is highly appealing because the impact of interpersonal synchronization seems to be immediate (e.g., Anshel \& Kipper, 1988; Cirelli et al., 2014; Hu et al., 2017; Reddish et al., 2013; Stupacher et al., 2017), and some types of antisocial behaviors such as aggression may arise at unpredictable times and require fast intervention. Helping people to manage the transient antisocial consequences of frustration with a fast response would, thus, be an important step in regulating daily interactions and preventing interpersonal escalades of aggression.

The main hypothesis of the present study was that interpersonal synchronization counteracts the antisocial outcomes of frustration. To that end, we invited a group of young, healthy participants to perform two joint tasks - synchronization versus non- 
synchronization. Each task was performed twice, with two different levels of induced frustration (high vs. low). We chose to make participants interact with a virtual partner since this allows controlling for potentially confounding interpersonal variables (Wiltermuth \& Heath, 2009). After each joint task, participants were measured for prosociality levels. At the end of the experiment, participants rated the perceived level of frustration during each task. We predicted that (1) induced frustration would yield decreases in prosociality in the non-synchronization task (frustration leading to aggression), but not (or to a lesser extent) in the synchronization task.

A secondary hypothesis of the present study related to methodological issues. We decided to use both explicit and implicit measures of prosociality, that is, tools that either favored participants' awareness of the instrument's purpose (explicit) or not (implicit). We did this to control for social desirability effects, which tend to be present in explicit measures such as self-report questionnaires (Saroglou et al., 2005). Measuring the participant's prosociality level unbeknownst to him/her is not straightforward: laboratory situations or games such as exposing participants to someone in need for help (Cirelli et al., 2014) or asking participants how much money they want to donate to someone else (e.g., dictator game, Bardsley, 2008; Eckel \& Grossman, 1996) capitalize on actions rather than self-reports and are, thus, less prone to social desirability or self-interpretation biases. Nevertheless, even these measures may yield artifacts (Bardsley, 2008) and, critically, they are difficult to implement in a repeatedmeasures design such as ours because participants may easily become suspicious of the experiment's goal. Facing this, we created and validated our own implicit measure of prosociality, which we named Interpersonal Trust Test (ITT). The ITT was designed to measure interpersonal trust in repeated-measures experimental designs, based on participants' interpretations of a set of different images depicting social interactions. We predicted that (2) the dissociation between synchronization and nonsynchronization tasks regarding the effects of frustration on prosociality would be more obvious when using implicit measures.

\section{Materials and Methods}

\section{Sample size}

Our effect of interest was the interaction between frustration and task (synchronization vs. non-synchronization) on prosociality. This was an untested hypothesis for which no findings were available in the literature. Therefore, as a heuristic, we focused on having enough power to capture the simple effect engaged in the interaction-frustration leading to decrease in prosociality in non-synchronization tasks. Available correlations between situational constraints (frustration) and aggression point to effect sizes (correlations) of .36 (Hershcovis et al., 2007). For this effect size, critical alpha of .05 and power of .80, G* power analyses (Faul et al., 2007) indicate a minimum sample size of 46 participants. 


\section{Participants}

Fifty college students took part in the experiment in exchange for course credits. Two of these were excluded due to technical errors leading to missing data, leaving us with 48 participants (6 male, mean age in years $\pm S D=22.1 \pm 5.6$ ). None reported psychiatric, neurologic or motor problems. All had normal or corrected-to-normal vision. All signed informed consent according to the Declaration of Helsinki.

\section{Stimulus materials}

Stimuli consisted of four videos (length of $120 \mathrm{sec}$ ), one per experimental condition (low-frustration synchronization, high-frustration synchronization, low-frustration non-synchronization, high-frustration non-synchronization). Videos showed graphic animations of a bouncing ball over a white background, at 30 frames per second (see Supplementary materials). Though these were pre-made video animations, participants were told that they were watching the online result of a partner's commands. All videos were subjected to piloting in the context of the experimental task (see below), being redesigned along the process to optimize frustration contrasts.

In the joint synchronization task, participants were asked to tap along with a beat. To that end, they viewed two animations of a blue bouncing ball setting a beat (beat onset each time the ball hit the imaginary ground and crushed). In the low-frustration condition, beat length was based on regular cycles of $750 \mathrm{~ms}$, with light jitter inserted $(M \pm S D=749 \mathrm{~ms} \pm 65)$ to grant the impression of human performance. In the highfrustration condition, beat cycles were highly variable $(M \pm S D=813 \mathrm{~ms} \pm 538)$. To increase the contrast between low- and high-frustration conditions, low-frustration videos had added sound (beep each time the ball hit the imaginary ground), while highfrustration ones were mute.

In the joint non-synchronization task, participants' task was to respond to changes in the color of an animated bouncing ball. The timing of the bouncing ball was irregular (same as in the high-frustration synchronization) to discourage any synchronization behavior, but the color of the ball kept changing (blue, black, green, yellow, or red). In the low-frustration version, there were few changes ( $n=31$, average change rate $=1$ every $3.87 \mathrm{sec})$. In the high-frustration condition, changes were significantly faster $(n=$ 230, average change rate $=1$ every $520 \mathrm{~ms}$ ). Changes were inserted at irregular time intervals. Paralleling synchronization stimuli, the low-frustration version included sound, while the high-frustration version did not.

\section{Instruments}

To obtain explicit measures of prosociality after each joint task, we used a standardized self-report questionnaire of altruism (Loureiro \& Lima, 2009). Among the available prosociality instruments validated for the Portuguese population, the AAS has the advantage of having been created and validated for adults, in contrast, for instance, with 
the Portuguese version of the Prosocial Tendencies Measure (PTM-R; Carlo et al., 2003), which has been validated only for early adolescents (Simões \& Calheiros, 2016). Together with the AAS, we ran an implicit experimental task of interpersonal trust (Implicit Trust Test, ITT), a questionnaire created and validated by the authors of this paper. Validation was carried out on a sample of 195 participants (52 male; 84 undergraduates, 78 graduates, 32 masters; one $\mathrm{PhD}$; Mean age $+S D=27.64 \pm 10.76$ ), who filled in both AAS and ITT in a single online survey.

Altruistic Attitudes Scale (AAS): The Altruistic Attitudes Scale is a self-report questionnaire that was developed and validated for the Portuguese population (Loureiro \& Lima, 2009). It contains 12 items, divided into three subscales/components that have been validated by confirmatory factorial analyses. These three components mirror theoretical views on the multidimensionality of altruistic attitudes and correspond to behavioral ("how often do you do the following"), cognitive ("how much do you agree with the following"), and affective ("how would you feel if you did the following," see Appendix 1, A1) items (four items per component). Participants respond using a 5point scale. The scale discriminates between volunteers and non-volunteers, and its components have internal consistency values (Cronbach's alpha) of .65 (cognitive), .81 (affective), and .70 (behavioral, .79 for the complete scale). In the present study, we used only the four cognitive and the four affective items as experimental measures (see Procedure section), given that reports of one's behaviors (behavioral items) are unlikely to change according to experimental conditions and would be thus inadequate to capture the changes in prosociality expected to occur during the experiment. Two behavioral items were used as fillers. In our validation study, the subset of eight cognitive and affective items yielded an alpha value of .61.

Implicit Trust-Test: the ITT was designed to capture one form of prosocialitytrust - in an implicit manner. Because it comprised different items - ultimately working as different tasks - the ITT allowed us to measure the same construct across different trials of within-subjects experiments, in contrast, for instance, with the dictator game (Bardsley, 2008; Eckel \& Grossman, 1996). Test Items consist of ambiguous images from the Thematic Apperception Test (TAT; Murray, 1943) where more than one character is depicted. In the original TAT test, participants are asked to tell one story based on each ambiguous image. In the ITT, we used some of these images to induce a forced-choice response: below each image, there are two possible interpretations - one that implies trust in one of the characters (i.e., the belief that s/he holds virtue or good intentions) and another implying mistrust (the character is viewed as dishonest, nonempathic, selfish, etc., see Appendix 1, A2). Participants are asked to pick one option among these two. Ambiguous test materials such as those presented in TAT images may reveal one person's patterns of thought, attitudes, observational capacity, and emotional responses (Gieser \& Stein, 1999), often providing information about a person's approach to interpersonal events (Ackerman et al., 2014).

Besides test items, there are filler items - images for which both options imply trust or mistrust. The purpose of filler items is to counteract participants' speculations that questions are about choosing between good and bad. The original version of this test 
was composed of eight test items and five fillers. We ran a validation study to maximize the internal consistency of items - which would be crucial to validate our repeatedmeasures approach, and also to get an idea of the extent to which the test captured prosocial dimensions. Following validation, we selected four test items and kept the five fillers.

The four test items were selected based on factorial analysis and reliability computations. Given that ITT items were binary, we based these computations on the polychoric correlation matrix (Starkweather, 2014) as implemented in the "polycor" package from R (Fox, 2019). Based on a one-factor analysis, we selected four ITT items with factor loadings above 0.3 (Yong \& Pearce, 2013): ITT 2: 0.665; ITT 4: 0.954; ITT 6: 0.532; ITT 7: 0.311 (see Appendix A, A2).

Means and standard deviations for AAS and ITT scores $(n=195)$ are shown in Table 1. ASS scores per participant were summed across the eight items (each response from 1 to 5) and divided by eight. Responses to ITT were coded as 1 when they matched the trust response option and 0 for the mistrust response. Scores were summed across the four items and divided by four. While schooling showed no effects on AAS or ITT scores $(p=.82 / .42)$, on AAS gender, it had an effect (Table 1) with women showing significantly higher scores. ITT showed no gender effects, perhaps reflecting reduced social-expectation influences compared to AAS (women expected to be more "sensitive" or altruistic). Age correlated significantly with ITT scores $(r=.15, p=.037)$ and marginally with AAS $(r=.14, p=.055)$, in line with evidence of age-related increases in prosociality (Matsumoto et al., 2016).

The reliability score (alpha based on polychoric correlation, see Gadermann et al., 2014) for the four-item subset was .78. ITT scores correlated significantly with AAS scores $(r=.315, p<.001$, Figure 1$)$, showing satisfactory convergent validity.

Post-experimental frustration questionnaire: This 5-point scale questionnaire was made up of two questions, both referring to each of the four moments when participants were performing a joint task. The questions were (1) How irritated were you? (2) How motivated were you to move on? For scoring purposes, responses to question (2) were reverted ( 1 becomes 5, 2 becomes 4 ) so that increasing scores had the same meaning of increased frustration. We chose a mood-related question (irritation) in line with other studies in this area (Gallucci et al., 2020). Since we may think of frustration as a concept that goes beyond mood, and as a state related to lack of motivation (Amsel \& Roussel, 1952), we added lack of motivation as a dimension of frustration.

Table I. Altruistic Attitudes Scale (AAS, range I-5) and Interpersonal Trust (ITT, range 0-I) scores per gender.

\begin{tabular}{lllll}
\hline & $\begin{array}{l}\text { Women } \\
M+S D\end{array}$ & Men & Total & Gender Effects \\
\hline AAS & $4.25+0.41$ & $4.07+0.47$ & $4.20+0.47$ & $t(193)=2.52, p=.012^{*}$ \\
ITT & $0.51+0.31$ & $0.43+0.30$ & $0.49+0.31$ & $t(193)=1.63, p=.105$ \\
\hline
\end{tabular}




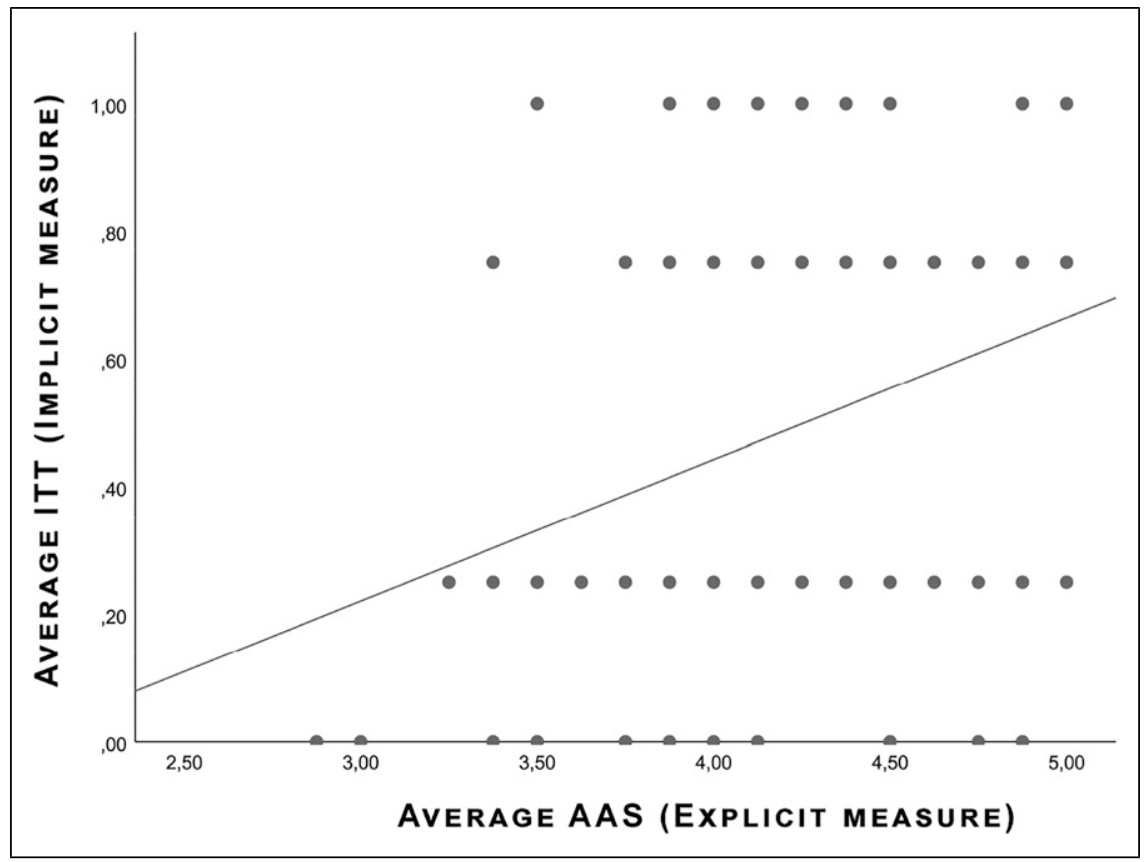

Figure I. Correlation between Altruistic Attitudes Scale (AAS, explicit measure of prosociality) and Interpersonal Trust Test (ITT, implicit measure of prosociality) scores in the validation study $(n=195)$.

\section{Procedure}

Participants were told they would take part in an experiment on "how people behave when performing joint tasks." Besides performing joint tasks, they should "provide their opinion on matters related to interpersonal relations" (reference to prosociality measures). When they entered the lab, the experimental partner was presented to them and then taken to an adjacent room with a non-transparent glass door and a computer connected to the experimental computer by means of visible cables. Participants were told that the computers were connected such that the actions of the partner would be visible on the participant's computer, and that they should follow the partner's commands at a distance. The experimenter remained in the main room, back turned on the participant, and asked the partner "can we start?" every time a new joint task began.

The experiment started with filler questions from the altruism questionnaire $(n=2)$ and from the Implicit Trust Test ( $n=1$, see Figure 2). The purpose of these initial filler questions was to divert participants' attention from our intention of measuring post-task prosociality levels. Participants then performed the four joint tasks: in the 


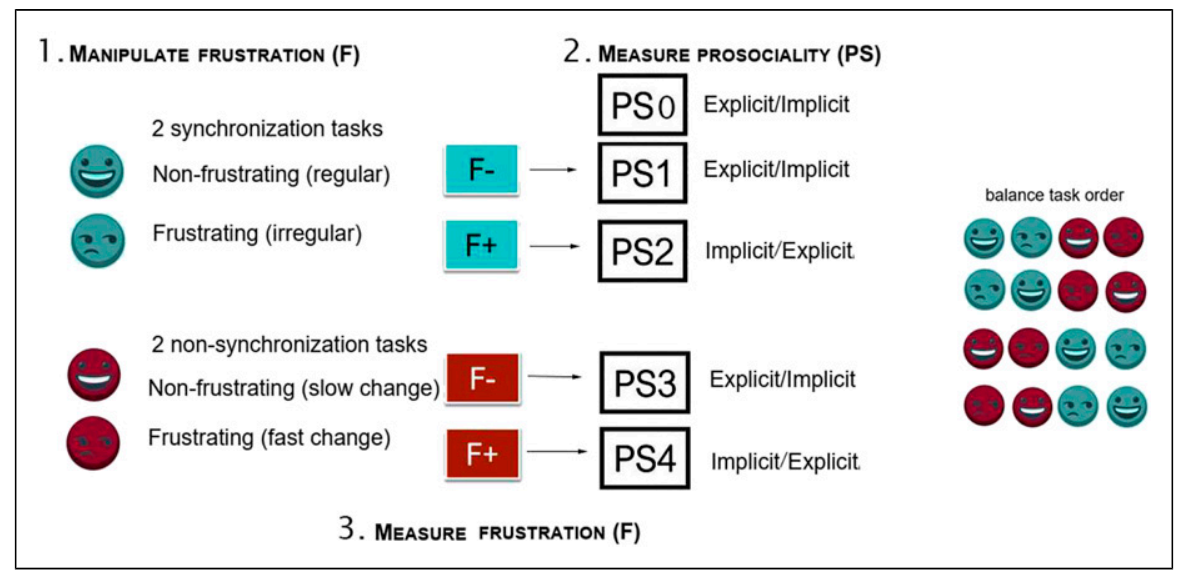

Figure 2. Diagram of the experimental procedure $(\mathrm{F}+/-=$ high/low frustration; $P S=$ prosociality measures: PSO = filler prosociality question).

synchronization task, they were asked to tap on a drum pad every time they saw the ball hitting the ground; in the non-synchronization task, they were asked to tap on the section of the drum pad containing the new color every time the ball changed colors. After each joint task, we measured prosociality using explicit measures (two AAS items, one cognitive and the other affective, see Appendix 1) and implicit ones (one ITT test item, coupled with a filler item).

Video stimuli and prosociality-related questions were all presented in the same eprime (https://pstnet.com/) script. Tests of prosociality followed the same order across all participants, while the order of joint tasks was counterbalanced (four orders, 12 participants each). This granted that the association between tasks and specific prosociality questions was not constant. The order of the two prosociality tests (explicit vs. implicit) was balanced across tasks.

At the end of the experiment, we asked participants what they thought the specific goal of the experiment was. None was aware of it. They then rated their levels of frustration in each task, considering both their state of irritation and motivation to move on. To rule out the possibility that the experimental session induced negative mood changes, we asked participants how happy and calm they were (5-point scale) before and after the experiment. After the experiment, participants were debriefed on the study's specific goals. 


\section{Analysis}

For each task (synchronization and non-synchronization), we computed differences between the high-frustration version and the low-frustration one concerning (a) perceived levels of irritation and lack of motivation (change in frustration), (b) AAS scores (change in explicit prosociality), and (c) ITT scores (change in implicit prosociality). Concerning (a), we found a significant correlation between changes in irritation and changes in motivation $(r=-.31, p=.002)$, and thus, we averaged these two dimensions of frustration into a single increase-in-frustration score.

We compared changes in perceived frustration across tasks using paired samples t-tests. We then used linear mixed-effects models as implemented in the lme package (Bates et al., 2014) for R (R Core Team, 2019) to determine the effects of change in frustration (continuous variable) $\times$ task (synchronization vs. non-synchronization) on changes in AAS scores (explicit measure of prosociality) and ITT scores (implicit). Following comparisons between one model with fixed factors only (change in frustration * task) and another that also included participants a random factor (random intercepts), we chose the latter for its improved fit $(p<.001)$. Thus, we had a two-level hierarchical model where task-related conditions (synchronization vs. non-synchronization, level 1) were nested within participants (level 2). The model was run twice-once for explicit prosociality (AAS) as dependent variable and then for implicit prosociality (ITT).

Significant interactions were followed by post-hoc analyses of the effects of changein-frustration per task, using a model containing only the interaction term (change in frustration:task) as fixed effect, and participants as random intercepts. Alpha levels were set to .05 . For the purpose of power analysis and effect size considerations, we considered both the unstandardized slope (Betas, presented in Table 2) and the corresponding standardized values.

Table 2. Predictors of change in prosociality (explicit, AAS, and implicit, ITT).

\begin{tabular}{|c|c|c|c|c|c|c|}
\hline \multirow[b]{2}{*}{ Fixed Parts } & \multicolumn{3}{|c|}{$\begin{array}{l}\text { Change in AAS (Explicit } \\
\text { Prosociality) }\end{array}$} & \multicolumn{3}{|c|}{$\begin{array}{l}\text { Change in ITT (Implicit } \\
\text { Prosociality) }\end{array}$} \\
\hline & Beta & SE & $t, p$ & Beta & SE & $t, p$ \\
\hline Change in frustration & -0.156 & 0.238 & $-0.658, .512$ & 0.100 & 0.096 & $1.037, .303$ \\
\hline Task & -0.104 & 0.494 & $-0.211, .834$ & 0.064 & 0.168 & $0.380, .705$ \\
\hline Change in frustration $\times$ task & 0.176 & 0.323 & $0.544, .588$ & -0.273 & 0.113 & $-2.415, .019 *$ \\
\hline \multicolumn{7}{|l|}{ Random parts } \\
\hline Variance & \multicolumn{3}{|l|}{2.499} & \multicolumn{3}{|l|}{0.229} \\
\hline SD & \multicolumn{3}{|l|}{1.581} & \multicolumn{3}{|l|}{0.478} \\
\hline${ }^{\mathrm{a}} \mathrm{R}^{2} \mathrm{~m} / \mathrm{R}^{2} \mathrm{c}$ & \multicolumn{3}{|c|}{$.0009 / .0009$} & \multicolumn{3}{|c|}{$.0507 / .6888$} \\
\hline Observations & \multicolumn{3}{|l|}{96} & \multicolumn{3}{|l|}{96} \\
\hline
\end{tabular}

${ }^{a} R^{2} m=$ Marginal $R^{2}$ (variance explained by fixed factors); $R^{2} c=$ Conditional $R^{2}$ (variance explained by fixed and random factors). 


\section{Results}

\section{Frustration rates per task}

Although non-synchronization generated larger increases in frustration $(M \pm S D=1.15$ $\pm 0.85)$ than synchronization tasks $(M \pm S D=0.60 \pm 0.96 ; t(47)=5.45, p<.001$; Figure 3), frustration rates for high-frustration versions $(\mathrm{F}+)$ were significantly higher than those for low-frustration versions $(\mathrm{F}-)$ in both tasks (synchronization: $t(47)=$ 4.32, $p<.001, d=0.72$; non-synchronization $(t(47)=10.51, p<.001, d=2.13)$, indicating that the high frustration versions increased frustration in both types of activities.

\section{Change in frustration as predictor of change in prosociality}

Changes in AAS (explicit prosociality) were not predicted by changes in frustration, task, or the interaction between the two (Table 2, Figure 4). However, we saw a significant change in frustration $\times$ task interaction on changes in ITT (implicit prosociality, Table 2, Figure 5). The standardized slope (effect size) of this interaction was -.349 , pointing to a medium effect size (Acock, 2014).

Concerning the effect size for the whole model run for ITT, the variance explained by fixed factors (marginal $\mathrm{R}^{2}=.05$ ) increased dramatically when random factors were

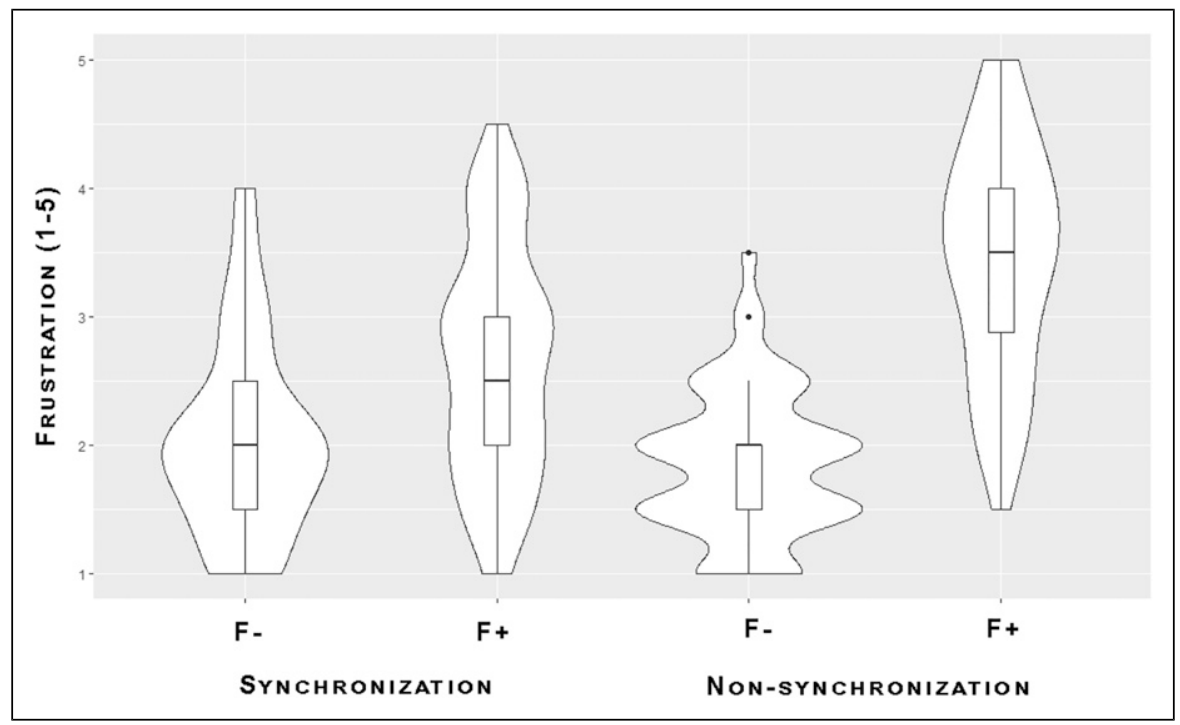

Figure 3. Frustration rates per task. Boxes indicate median and interquartile ranges; outer shapes indicate probability densities (data distribution), smoothed by a kernel density estimator. 


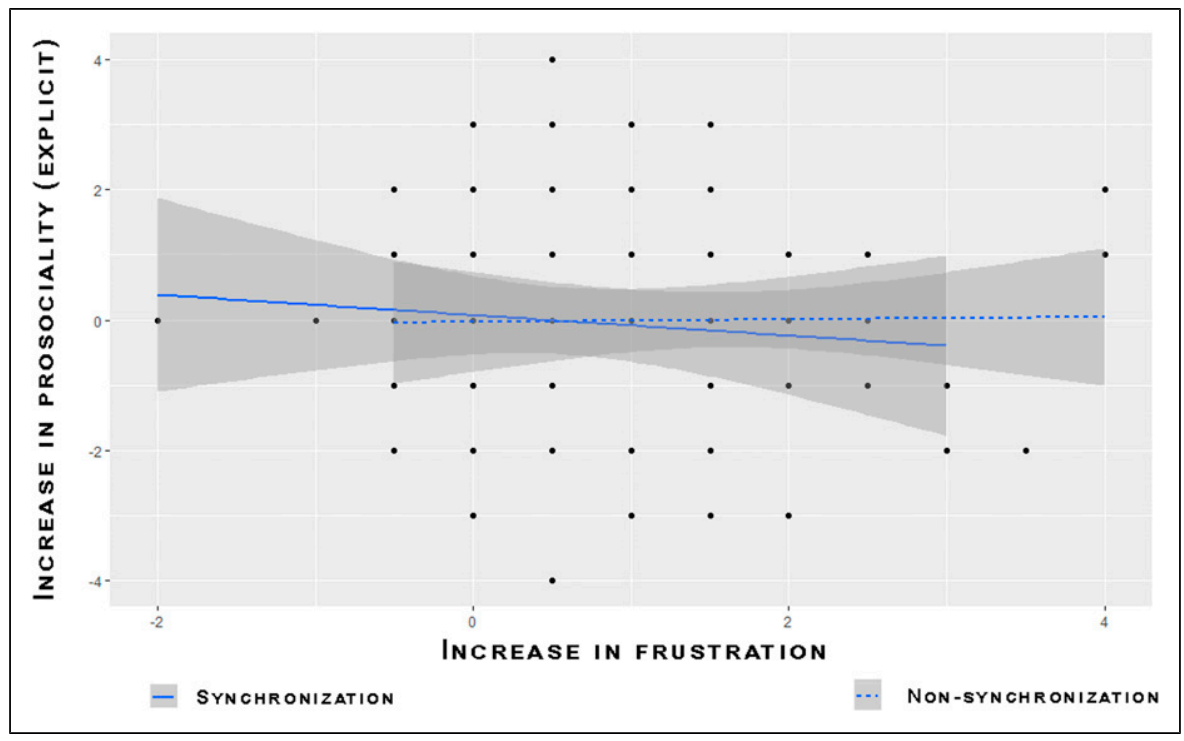

Figure 4. Changes in explicit prosociality (measured with AAS) as a function of changes in frustration across synchronization versus non-synchronization tasks. Changes in frustration had no effects in any task.

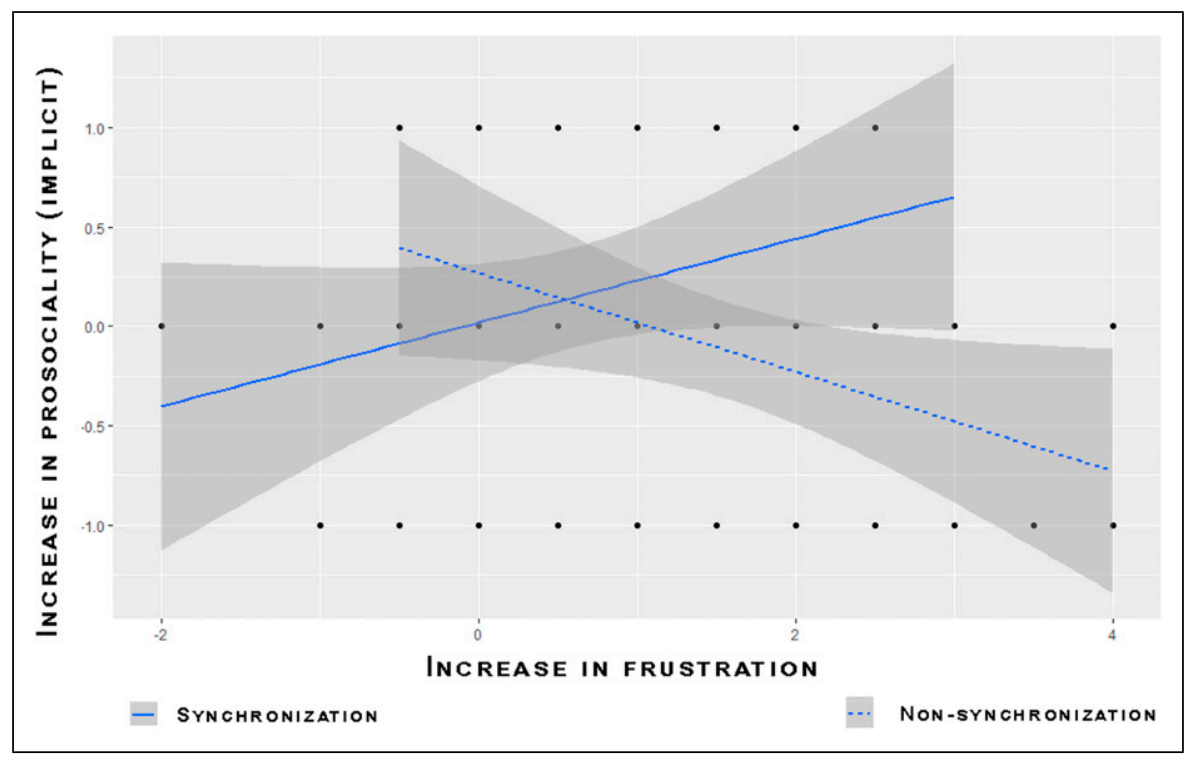

Figure 5. Changes in implicit prosociality (measured with ITT) as a function of changes in frustration across synchronization versus non-synchronization tasks. Increases in frustration decreased prosociality levels after non-synchronization tasks, but not after synchronization. 
added (conditional $\mathrm{R}^{2}=.688$, see Nakagawa \& Schielzeth, 2013). This is line with the model comparisons we did (with vs. without random factors, see 2.6) and highlights the importance of individual characteristics in the effects under analysis.

Post-hoc analyses of the frustration $\times$ task interaction on ITT showed that implicit prosociality decreased with increases in frustration for the non-synchronization task $(B=-0.15, S E=0.06, t=-2.51, p=.014$, standardized $B=-0.32)$ but not for synchronization $(B=0.09, S E=0.09, t=0.99, p=.032$, standardized $B=0.02)$.

\section{Experiment-induced mood changes}

Participants' levels of calm and happiness at the beginning of the experiment (happiness, $M \pm S D=3.85+0.61$; calm, $3.56 \pm 1.00)$ did not change significantly when the session was over (happiness, $\mathrm{M} \pm \mathrm{SD}=3.98 \pm 0.66, t(47)=1.95, p=.057$; calm, $3.81 \pm$ $0.86, t(47)=1.57, p=.122)$.

\section{Power analyses}

The core result of this study was the significant interaction between frustration and task on prosociality, which was indexed by an effect size (Beta, or slope) of -.27 . The first power-related question that emerges concerns the likelihood of replicating this interaction in a new study. Using the SIMR package for R (Green \& MacLeod, 2016), we did a post-hoc power analysis based on simulations ( 200 simulations per run). First, we determined the probability of capturing the frustration $\times$ task interaction in a new study with the current sample size $(n=48)$. Based on the observed value of the interaction slope $(B=-.27)$, the analysis pointed to $66.5 \%$ power $(C I 95 \%: 59.50-73.00 \%)$. By running a power curve, we then estimated how many participants we would need to reach $80 \%$ power. The results are presented in Figure 6, and they point to a sample of 70 participants. From this viewpoint, it is wise to look at our findings as preliminary ones (see discussion).

As for the simple effects of frustration on prosociality (one effect per task), $G^{*}$ Power sensitivity analyses using standardized slopes as effect sizes for simple regression indicated that the minimum detectable significant slope in our experiment would be $(-.342)$. We did detect a similar standardized slope in the non-synchronization task $(-.322)$, while the slope for synchronization (.02) remained non-significant. The interesting thing about frustration effects in the synchronization task was that even though null, they were opposite to frustration effects in non-synchronization. Therefore, if the small effect size in synchronization is real and we did not capture it as significant due to sample size issues, this would mean that synchronization may even reverse the antisocial effects of frustration, rather than simply attenuate them. 


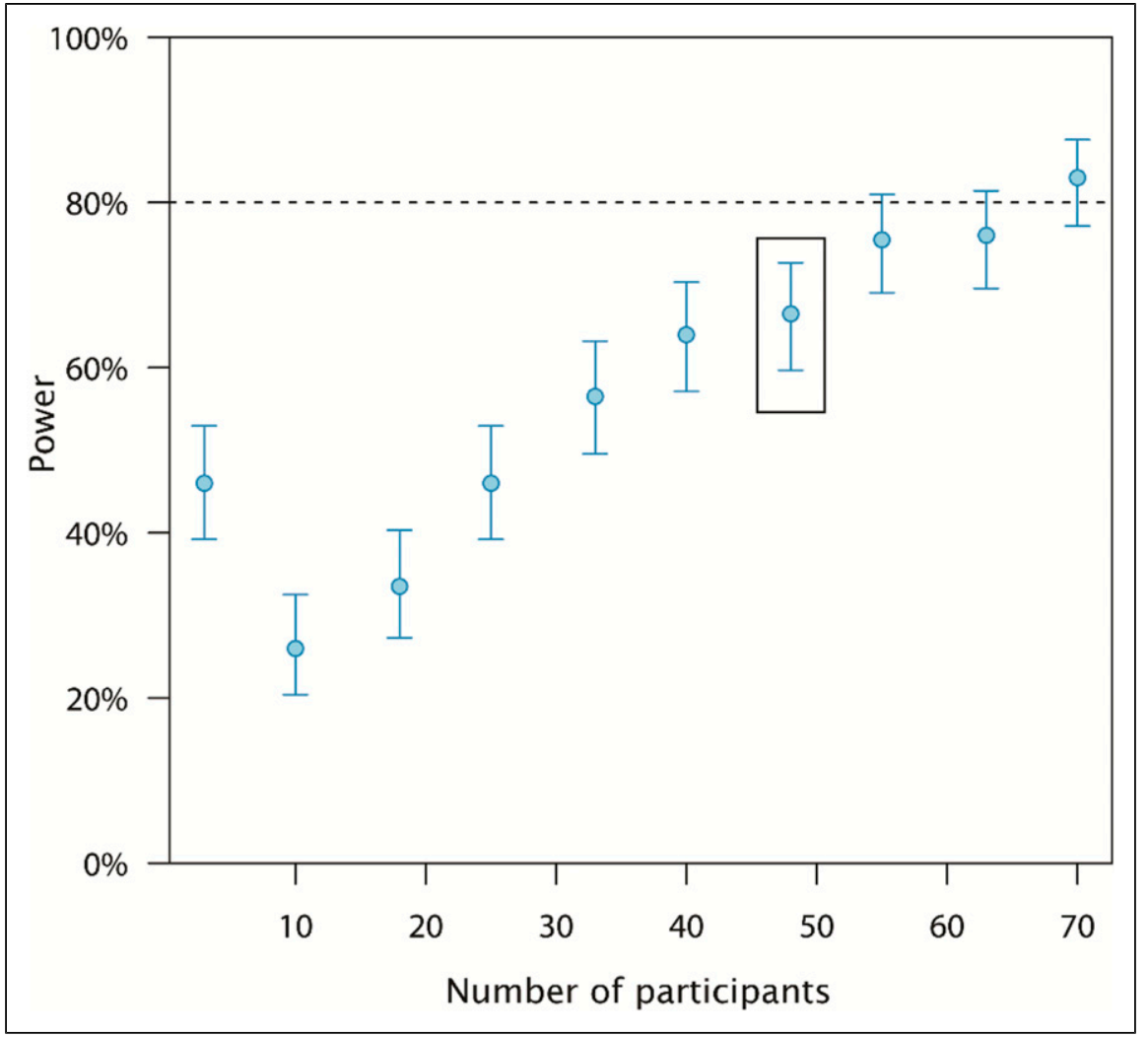

Figure 6. Power estimates according to sample size. Power obtained with the current sample $(n=48)$ is highlighted. Vertical bars represent confidence intervals of $95 \%$.

\section{Discussion}

We wanted to test one manifestation of interpersonal synchronization effects on prosociality, namely, whether the well-known antisocial outcomes (decrease in prosociality) of increased frustration are attenuated when frustration occurs in the context of a synchronization task. To that end, we asked participants to perform synchronization and non-synchronization tasks under different levels of induced frustration, and we compared the impact of changes in frustration on changes in prosociality across the two tasks. In the non-synchronization task, increases in frustration led to decreases in prosociality, in line with the classic frustration-aggression hypothesis (Blair, 2001; Giacalone \& Greenberg, 1997; Miller, 1941). Supporting our hypothesis, increases in frustration occurring during synchronization tasks did not have such impact. Thus, although participants felt increasingly frustrated as synchronization with the partner became more difficult, such increased frustration did not make them less prosocial. 
What do our findings add to the growing evidence that interpersonal synchronization enhances prosociality? The answer is that they point to the possibility of counteracting sudden negative changes in prosociality: while previous studies focused on the possibility of enhancing basal levels of prosociality (i.e., participants' default state as they entered the experiment), we showed that interpersonal synchronization can act upon temporary antisocial states caused by external (frustrating) circumstances - what Blair coined as reactive aggression (Blair, 2001). The potential applications of this possibility are numerous, particularly in the fields of social deviance and organizational behavior, where the concern with continuous regulation of interpersonal interactions is prioritary. Our effect of interest - the interaction between task and change in frustration-showed a medium effect size (standardized slope of - .349), which may be considered an effect of practical interest. Thus, interpersonal synchronization seems to create non-negligible changes in frustration-related aggression, which may make a difference in people's lives.

A second important finding of our study was that implicit, but not explicit measures of prosociality were able to capture fast changes in prosociality levels, pointing to the benefits of implicit measures in this research domain.

Our study raised a novel hypothesis that expands current knowledge on the prosocial effects of interpersonal synchronization, and our preliminary findings seem to have provided support to it. While taking this step forward, we did not look at the methodological choices we made in our study as closed solutions. Therefore, future work is needed regarding the appropriateness of our choices. One priority is, of course, increasing power by using larger sample sizes, following the numbers we provided in the results based on power simulations (see 3.4). Beyond that the validity of including motivation in our frustration measures - a non-universal approach - is one issue to consider in followup studies. Another one concerns the exact nature of the prosociality-related construct that is being captured by our implicit measure and its relation to other prosociality dimensions: for instance, how does the dimension we called interpersonal trust relates to prosocial behaviors like help or cooperation? Finally, the potential moderating role of individual characteristics on the effect we saw is another issue that invites future developments. As we saw from the contribution of participants as random factors in explaining variance in prosociality responses, individual characteristics seem to make a large difference. Specifying which individual characteristics play a major moderating role in the protective effects of synchronization on frustration-related antisociality is, thus, an open challenge. Analyzing gender differences regarding the mechanisms under analysis (see, e.g., Gallucci et al., 2020) could be a priority since our unbalanced sample (6 men) did not allow us to do it in the present study. Besides gender, personality traits (e.g., emotional stability) could also be important targets.

Concerning the mechanisms subtending the link between interpersonal synchronization and prosociality, our findings did not allow us to move far beyond current hypotheses (increased perception of the other person during synchronization leading to empathy and, consequently, to prosociality): the particular scenario we examined (counteracting the antisocial effects of frustration with interpersonal synchronization) is compatible with the 
hypothesized mechanisms. Nevertheless, the fact that the effects of frustration-a circumstance where loss of power is usually engaged - may be counteracted with interpersonal synchronization allows us to speculate on the possible links between synchronization and interpersonal power: could it be that the prosociality that is added with interpersonal synchronization arises from a feeling of power/domination over the other person? Could it be that the attentional unit and/or the ability to anticipate the other person's gestures empowers the individual, instead of, or along with a sense of empathy? This would be consistent with evidence that interpersonal synchronization activates the reward system (Kokal et al., 2011), and it could be investigated with manipulations of power roles during synchronization tasks (effect of leading vs. following a partner).

In sum, our study highlighted a new facet of the effects of interpersonal synchronization on prosociality, thus strengthening and expanding the idea that prosociality can be modeled by circumstances. Considering that prosociality is a factor of happiness (Aknin et al., 2015), well-being (Helliwell et al., 2017), and economic development (Torsvik, 2000), and that - unlike genetics or culture-circumstances can be shaped, continuing the investigation of situational influences that promote prosocial behaviors may be greatly valuable in the years to come.

\section{Appendix I}

\section{Instruments}

\section{Al. Altruistic Attitudes Scale}

The 12 original items are presented below. Q(x)s indicate the order that selected items were presented in the experiment ( 2 items after each joint task; $\mathrm{q} 0 \mathrm{a}$ and $\mathrm{q} 0 \mathrm{~b}$ presented at the beginning of the experiment; * indicate inverted questions, were higher scores mean less altruism).

Cognitive subscale. Please indicate how much you agree with the following statements: Disagree (1) Agree (5)

01. I think that each of us must take care of one's own life (q1*)

02. I think it is important to respect other people's feelings (q3)

03 . Those who are altruistic always regret it later $\left(\mathrm{q} 5^{*}\right)$

04. The benefits of altruism do not compensate for the sacrifices (q7*)

Affect subscale. Think how you would feel if you did the following:

Very bad (1) Very good (5)

05. Take care of someone without expecting any reward (q2)

06 . Assist your family and friends without expecting anything in return (q4)

07. Help an institution without having been asked to do so (q6) 
08. Bewilling to sacrífice yourself to help someone (q8)

Behavioral scale. How often do you do the following:

Never (1) Quite often (5)

09. Point out street directions to a stranger

10. Keep the elevator's door open so that someone can come in (q0a)

11. Give away to someone my place in a supermarket queue (q0b)

12. Help someone at work in performing a task

\section{A2. Implicit Trust Test}

Underlined items were the ones selected after validation.

Choose the option that best fits what you see in the image:

ITT FI (two positive options). Image description: An adolescent boy looks straight out of the picture. The barrel of a rifle is visible at one side, and in the background is a dim scene of a surgical operation, like a reverie-image.

Question

The young man is a medical student. He is feeling proud because

The surgery is going well $(+)$

He devised an innovative procedure $(+)$

ITT F2 (two negative options). Image description: A gray-haired man is looking at a younger man who is sullenly staring into space.

Question. The older man is

Questioning his son's honesty (-)

Manipulating his son (-)

\section{A3. ITT I}

Image description: A young woman is standing with a downcast head buried in her arm. Behind her is the figure of a man lying in bed.

\section{Question}

The man is drunk. The woman is crying because

She is worried about him $(+)$

She sees no future in their relation $(-)$

ITT2. Image description: A young man looks downward while standing beside a taller, older man. The older man stares into space. 
Question. The son committed a crime. His father Is trying to find a way of protecting his son $(+)$

Is afraid the situation brings trouble to himself and his family $(-)$

ITT 3. Image description: A man is standing in the foreground with his head in his arms. In the background is a woman lying in bed.

Question. The girl was raped by a criminal. The man covers his eyes because He could have done something to save the girl, but he did not do it for selfishness (-) He is shy to see the girl naked $(+)$

ITT 4. Image description: Portrait of a young woman. A weird old woman with a shawl over her head is grimacing in the background.

Question. The older woman is about to give good advice to the younger person $(+)$ snoop into the younger person's life $(-)$

ITT F3 (both positive). Image description: In the foreground is a young woman with books in her hands; in the background, a man is working in the field and an older woman is looking on.

Question. The young woman is planning

To help the workers with money $(+)$

To help the workers learning to read $(+)$

ITT 5. Image description: A woman has her hands squeezed around the throat of a man whom she appears to be pushing backwards across the bannister of a stairway.

Question. The woman is worried because

The man fell down the stairs and she is afraid the police will accuse her of murder (-) She fears for the man's life $(+)$

ITT F4 (both negative)

Image description: A gaunt man with clinched hands is standing among gravestones.

Question. The man

Helped murdering someone (-)

Is planning on murdering someone $(-)$

ITT 6. Image description: A middle-aged woman is standing on the threshold of a halfopen door looking into a room. 
Question. The maid is

Checking whether the path is free for her to steal money (-)

Checking whether the lady is fine $(+)$

ITT F5 (both positive). Image description: Inside a room, a man kneels and looks through a glass door with his left hand over a cannon.

Question. The man is

Defending the safety of his mother $(+)$

Defending the safety of his children $(+)$

ITT 7. Image description: An older woman is sitting on a sofa beside a girl, speaking or reading to her. The girl, who has a doll on her lap, is looking far away.

Question. The lady is reading the child a story with enthusiasm. The child Is planning to tell the story to her friends $(+)$

Is bored and wants to run away $(-)$

ITT 8. Image description: A short elderly woman stands with her back turned to a tall young man. The latter is looking downward with a perplexed expression.

Question. The man is apprehensive because

He wants to do business with the old woman but she is not interested (-)

The old woman told him she is sick $(+)$

\section{A3. Experimental items}

Task I. AAS 01-AAS 05-ITTF2-ITT2

Task 2. ITT 4-ITTf3-AAS 02-AAS 06

Task 3. AAS 03-AAS 07-ITTf4-ITT6

Task 4. ITTF5-ITT7-AAS4-AAS8

\section{Acknowledgments}

We are grateful to Fernanda Siqueira, Júlia Cipriano, and Ana Mesquita for their help with data collection.

\section{Declaration of Conflicting Interests}

The author(s) declared no potential conflicts of interest with respect to the research, authorship, and/or publication of this article. 


\section{Funding}

The author(s) disclosed receipt of the following financial support for the research, authorship, and/or publication of this article: This work was funded by the Portuguese Science Foundation (CPUP FCT UIDB/00050/2020).

\section{Ethical Approval}

The authors declare no conflict of interest. Participants were healthy adults who signed informed consent for behavioral data collection according to the Declaration of Helsinki. Participants' well-being during the experiment was carefully monitored during the experiment (see Methods and Results), and they were free to quit at any time (which never happened). Under these circumstances, approval by an ethical committee is not mandatory in our institution.

\section{Data Availability}

The datasets generated and analyzed during the current study as well as the analysis code and supplementary materials are available in the OSF repository (https://osf.io/nmvb4/?view_only= 364e11f3fffd454bac23b7d5cfd062ac).

\section{ORCID iD}

Susana Silva (D) https://orcid.org/0000-0003-2240-1828

\section{References}

Ackerman, S. J., Lewis, K. C., \& Taylor, A. E. (2014). The thematic apperception test: A performance-based assessment technique. In Personality assessment (pp. 383-412). Routledge. https://doi.org/10.4324/9780203119143-15

Acock, A. C. (2014). A gentle introduction to stata (4th ed.): Stata Press. 9781597181426.

Ahmed, A., \& Salas, O. (2013). Religious context and prosociality: An experimental study from valparaíso, Chile. Journal for the Scientific Study of Religion, 52(3), 627-637. https://doi. org/10.1111/jssr.12045

Aknin, L. B., Broesch, T., Hamlin, J. K., \& Van de Vondervoort, J. W. (2015). Prosocial behavior leads to happiness in a small-scale rural society. Journal of Experimental Psychology: General, 144(4), 788-795. https://doi.org/10.1037/xge0000082

Amsel, A., \& Roussel, J. (1952). Motivational properties of frustration: I. effect on a running response of the addition of frustration to the motivational complex. Journal of Experimental Psychology, 43(5), 363-368. https://doi.org/10.1037/h0059393

Ando, J., \& Kawamoto, T. (2021). Genetic and environmental structure of altruism characterized by recipients in relation to personality. Medicina, 57(6), 593. https://doi.org/10.3390/ medicina57060593

Anshel, A., \& Kipper, D. A. (1988). The influence of group singing on trust and cooperation. Journal of Music Therapy, 25(3), 145-155. https://doi.org/10.1093/jmt/25.3.145

Arnone, M., \& Dantzer, R. (1980). Does frustration induce aggression in pigs? Applied Animal Ethology, 6(4), 351-362. https://doi.org/10.1016/0304-3762(80)90135-2 
Bardsley, N (2008). Dictator game giving: Altruism or artefact? Experimental Economics, 11(2), 122-133. https://doi.org/10.1007/s10683-007-9172-2

Bates, D., Mächler, M., Bolker, B., \& Walker, S. (2014). Fitting linear mixed-effects models using lme4. arXiv:1406.5823 [stat]. http://arxiv.org/abs/1406.5823

Batson, C. D., \& Powell, A. A. (2003). Altruism and prosocial behavior. In Handbook of psychology (pp. 463-484). American Cancer Society. https://doi.org/10.1002/0471264385. wei0519

Behrends, A., Müller, S., \& Dziobek, I. (2012). Moving in and out of synchrony: A concept for a new intervention fostering empathy through interactional movement and dance. The Arts in Psychotherapy, 39(2), 107-116. https://doi.org/10.1016/j.aip.2012.02.003

Bereczkei, T., Birkas, B., \& Kerekes, Z. (2010). The presence of others, prosocial traits, machiavellianism. Social Psychology, 41(4), 238-245. https://doi.org/10.1027/1864-9335/ a000032

Bernieri, F. J., Reznick, J. S., \& Rosenthal, R. (1988). Synchrony, pseudosynchrony, and dissynchrony: Measuring the entrainment process in mother-infant interactions. Journal of Personality and Social Psychology, 54(2), 243-253. https://doi.org/10.1037/0022-3514.54. 2.243

Blair, R. J. R. (2001). ADVANCES IN NEUROPSYCHIATRY: Neurocognitive models of aggression, the antisocial personality disorders, and psychopathy. Journal of Neurology, Neurosurgery \& Psychiatry, 71(6), 727-731. https://doi.org/10.1136/jnnp.71.6.727

Brief, A. P., \& Motowidlo, S. J. (1986). Prosocial organizational behaviors. Academy of Management Review, 11(4), 710-725. https://doi.org/10.5465/amr.1986.4283909

Cacioppo, S., Zhou, H., Monteleone, G., Majka, E. A., Quinn, K. A., Ball, A. B., Norman, G. J., Semin, G. R., \& Cacioppo, J. T. (2014). You are in sync with me: Neural correlates of interpersonal synchrony with a partner. Neuroscience, 277, 842-858. https://doi.org/10. 1016/j.neuroscience.2014.07.051

Carlo, G., Hausmann, A., Christiansen, S., \& Randall, B. A. (2003). Sociocognitive and behavioral correlates of a measure of prosocial tendencies for adolescents. The Journal of Early Adolescence, 23(1), 107-134. https://doi.org/10.1177/0272431602239132

Christian Cadenhead, A., \& Richman, C. L. (1996). The effects of interpersonal trust and group status on prosocial and aggressive behaviors. Social Behavior and Personality: an International Journal, 24(2), 169-184. https://doi.org/info:doi/10.2224/sbp.1996.24.2.169

Cirelli, L. K., Einarson, K. M., \& Trainor, L. J. (2014). Interpersonal synchrony increases prosocial behavior in infants. Developmental Science, 17(6), 1003-1011. https://doi.org/10. 1111/desc. 12193

Eckel, C. C., \& Grossman, P. J. (1996). Altruism in anonymous dictator games. Games and Economic Behavior, 16(2), 181-191. https://doi.org/10.1006/game.1996.0081

Ellis, L., Hoskin, A. W., \& Ratnasingam, M. (2018). Handbook of social status correlates. Academic Press

Fairhurst, M. T., Janata, P., \& Keller, P. E. (2013). Being and feeling in sync with an adaptive virtual partner: brain mechanisms underlying dynamic cooperativity. Cerebral Cortex, 23(11), 2592-2600. https://doi.org/10.1093/cercor/bhs243 
Faul, F., Erdfelder, E., Lang, A.-G., \& Buchner, A. (2007). G*Power 3: A flexible statistical power analysis program for the social, behavioral, and biomedical sciences. Behavior Research Methods, 39(2), 175-191. https://doi.org/10.3758/BF03193146

Foubert, K, Collins, T, \& De Backer, J (2017). Impaired maintenance of interpersonal synchronization in musical improvisations of patients with borderline personality disorder. Frontiers in Psychology, 8, 537. https://doi.org/10.3389/fpsyg.2017.00537

Fox, J. (2019). The 'polycor' package. Documentation available at CRAN 2014:. http://cran.rproject.org/web/packages/polycor/index.html

Gadermann, A., Guhn, M., \& Zumbo, B. D. (2014). Ordinal alpha. In A. C. Michalos (Ed), Encyclopedia of Quality of Life and Well-Being Research (pp. 4513-4515). Springer Netherlands. https://doi.org/10.1007/978-94-007-0753-5_2025

Gallucci, A., Riva, P., Romero Lauro, L. J., \& Bushman, B. J. (2020). Stimulating the ventrolateral prefrontal cortex (VLPFC) modulates frustration-induced aggression: A tDCS experiment. Brain Stimulation, 13(2), 302-309. https://doi.org/10.1016/j.brs.2019.10.015.

Giacalone, R. A., \& Greenberg, J. (1997). Antisocial behavior in organizations. SAGE

Gieser, L., \& Stein, M. I. (1999). Evocative images: The thematic apperception test and the art of projection. American Psychological Association. https://doi.org/10.1037/10334-000

Green, P., \& MacLeod, C. J. (2016). SIMR : An R package for power analysis of generalized linear mixed models by simulation. Methods in Ecology and Evolution, 7(4), 493-498. https://doi.org/10.1111/2041-210X.12504

Helliwell, J. F., Aknin, L. B., Shiplett, H., Huang, H., \& Wang, S. (2017). Social Capital and prosocial Behaviour as Sources of well-being (working paper N. 23761). National Bureau of Economic Research. https://doi.org/10.3386/w23761

Hershcovis, M. S., Turner, N., Barling, J., Arnold, K. A., Dupré, K. E., Inness, M., LeBlanc, M. M., \& Sivanathan, N. (2007). Predicting workplace aggression: A meta-analysis. Journal of Applied Psychology, 92(1), 228-238. https://doi.org/10.1037/0021-9010.92.1. 228

Hove, M. J., \& Risen, J. L. (2009). It's all in the timing: interpersonal synchrony increases affiliation. Social Cognition, 27(6), 949-960. https://doi.org/10.1521/soco.2009.27.6.949

Hu, Y., Hu, Y., Li, X., Pan, Y., \& Cheng, X. (2017). Brain-to-brain synchronization across two persons predicts mutual prosociality. Social Cognitive and Affective Neuroscience, 12(12), 1835-1844. https://doi.org/10.1093/scan/nsx118

Kaufman, A. (2004). Curbing innovation: How command technology limits network centric warfare. Argos Press

Keller, P. E., Novembre, G., \& Hove, M. J. (2014). Rhythm in joint action: psychological and neurophysiological mechanisms for real-time interpersonal coordination. Philosophical Transactions of the Royal Society B: Biological Sciences, 369(1658), 20130394. https://doi. org/10.1098/rstb.2013.0394

Keltner, D., Kogan, A., Piff, P. K., \& Saturn, S. R. (2014). The Sociocultural Appraisals, Values, and Emotions (SAVE) Framework of prosociality: core processes from Gene to meme. Annual Review of Psychology, 65(1), 425-460. https://doi.org/10.1146/annurev-psych010213-115054 
Kirschner, S., \& Tomasello, M. (2010). Joint music making promotes prosocial behavior in 4year-old childrenşs 3 斿. Evolution and Human Behavior, 31(5), 354-364. https://doi.org/ 10.1016/j.evolhumbehav.2010.04.004

Knafo-Noam, A., Uzefovsky, F., Israel, S., Davidov, M., \& Zahn-Waxler, C. (2015). The prosocial personality and its facets: Genetic and environmental architecture of motherreported behavior of 7-year-old twins. Frontiers in Psychology, 6, 112. https://doi.org/10. 3389/fpsyg.2015.00112

Knafo, A., \& Israel, S. (2010). Genetic and environmental influences on prosocial behavior. In Em Prosocial motives, emotions, and behavior: The better angels of our nature (pp. 149-167). American Psychological Association. https://doi.org/10.1037/12061-008

Knafo, A., \& Plomin, R. (2006). Parental discipline and affection and children's prosocial behavior: genetic and environmental links. Journal of Personality and Social Psychology, 90(1), 147-164. https://doi.org/10.1037/0022-3514.90.1.147

Koban, L., Ramamoorthy, A., \& Konvalinka, I. (2019). Why do we fall into sync with others? Interpersonal synchronization and the brain's optimization principle. Social Neuroscience, 14(1), 1-9. https://doi.org/10.1080/17470919.2017.1400463

Kokal, I., Engel, A., Kirschner, S., \& Keysers, C. (2011). Synchronized drumming enhances activity in the caudate and facilitates prosocial commitment - if the rhythm comes easily. PLOS ONE, 6(11), e27272. https://doi.org/10.1371/journal.pone.0027272

Launay, J., Dean, R. T., \& Bailes, F. (2013). Synchronization can influence trust following virtual interaction. Experimental Psychology, 60(1), 53-63. https://doi.org/10.1027/1618-3169/a000173

Lefevor, G. T., Fowers, B. J., Ahn, S., Lang, S. F., \& Cohen, L. M. (2017). To what degree do situational influences explain spontaneous helping behaviour? A meta-analysis. European Review of Social Psychology, 28(1), 227-256. https://doi.org/10.1080/10463283.2017.1367529

Loureiro, A., \& Lima, M. L. (2009). Escala de atitudes altruístas: Estudo de validação e fiabilidade. Laboratório de Psicologia, 7, 73-83.

Luria, G., Cnaan, R. A., \& Boehm, A. (2015). National culture and prosocial behaviors. Nonprofit and Voluntary Sector Quarterly, 44(5), 1041-1065. https://doi.org/10.1177/ 0899764014554456

Marsh, K. L., Richardson, M. J., \& Schmidt, R. C. (2009). Social connection through joint action and interpersonal coordination. Topics in Cognitive Science, 1(2), 320-339. https://doi.org/ 10.1111/j.1756-8765.2009.01022.x

Matsumoto, Y., Yamagishi, T., Li, Y., \& Kiyonari, T. (2016). Prosocial behavior increases with age across five economic games. PLOS ONE, 11(7), e0158671. https://doi.org/10.1371/ journal.pone.0158671

Miller, N. E. (1941). I. The frustration-aggression hypothesis. Psychological Review, 48(4), 337-342. https://doi.org/10.1037/h0055861

Murray, H. A. (1943). Thematic apperception test. Harvard University Press

Nakagawa, S., \& Schielzeth, H. (2013). A general and simple method for obtainingR2from generalized linear mixed-effects models. Methods in Ecology and Evolution, 4(2), 133-142. https://doi.org/10.1111/j.2041-210x.2012.00261.x

Padilla-Walker, L. M., \& Carlo, G. (2015). Prosocial development: A multidimensional approach. Oxford University Press 
R Core Team (2019). R: A language and environment for statistical computing: R Foundation for Statistical Computing. https://www.R-project.org/

Rand, DG, \& Kraft-Todd, GT (2014). Reflection does not undermine self-interested prosociality. Frontiers in Behavioral Neuroscience, 8, 300. https://doi.org/10.3389/fnbeh.2014.00300

Reddish, P., Fischer, R., \& Bulbulia, J. (2013). Let's dance together: synchrony, shared intentionality and cooperation. PLOS ONE, 8(8), e71182. https://doi.org/10.1371/journal. pone. 0071182

Rennung, M., \& Göritz, A. S. (2016). Prosocial consequences of interpersonal synchrony. Zeitschrift für Psychologie, 224(3), 168-189. https://doi.org/10.1027/2151-2604/a000252

Roederer, J. G. (1984). The search for a survival value of music. Music Perception, 1(3), 350-356. https://doi.org/10.2307/40285265

Rotenberg, K. J., Fox, C., Green, S., Ruderman, L., Slater, K., Stevens, K., \& Carlo, G. (2005). Construction and validation of a children's interpersonal trust belief scale. British Journal of Developmental Psychology, 23(2), 271-293. https://doi.org/10.1348/026151005X26192

Saroglou, V., Pichon, I., Trompette, L., Verschueren, M., \& Dernelle, R. (2005). Prosocial behavior and religion: New evidence based on projective measures and peer ratings. Journal for the Scientific Study of Religion, 44(3), 323-348. https://doi.org/10.1111/j.1468-5906.2005.00289.x

Simões, F, \& Calheiros, MM (2016). The relations between prosocial behaviors and selfregulation: evidences from the validation of the ptm-r for portuguese early adolescents. The Spanish Journal of Psychology, 19, E73. https://doi.org/10.1017/sjp.2016.70

Skoe, E. E. A., Cumberland, A., Eisenberg, N., Hansen, K., \& Perry, J. (2002). The influences of sex and gender-role identity on moral cognition and prosocial personality traits. Sex Roles, 46(9), 295-309. https://doi.org/10.1023/A:1020224512888

Starkweather, J. (2014). Factor analysis with binary items: A quick review with examples. Benchmarks RSS matters. http://it.unt.edu/benchmarks

Stupacher, J., Maes, P.-J., Witte, M., \& Wood, G. (2017). Music strengthens prosocial effects of interpersonal synchronization - if you move in time with the beat. Journal of Experimental Social Psychology, 72, 39-44. https://doi.org/10.1016/j.jesp.2017.04.007

Torsvik, G. (2000). Social capital and economic development. Rationality and Society, 12(4), 451-476. https://doi.org/10.1177/104346300012004005

van den Bos, W., Crone, E. A., Meuwese, R., \& Güroğlu, B. (2018). Social network cohesion in school classes promotes prosocial behavior. PLOS ONE, 13(4), e0194656. https://doi.org/ 10.1371/journal.pone.0194656

Walster, E., \& Piliavin, J. A. (1972). Equity and the innocent bystander. Journal of Social Issues, 28(3), 165-189. https://doi.org/10.1111/j.1540-4560.1972.tb00038.x

Wiltermuth, S. S., \& Heath, C. (2009). Synchrony and cooperation. Psychological Science, 20(1), 1-5. https://doi.org/10.1111/j.1467-9280.2008.02253.x

Yong, A. G., \& Pearce, S. (2013). A beginner's guide to factor Analysis: Focusing on exploratory factor analysis. Tutorials in Quantitative Methods for Psychology, 9(2), 79-94. https://doi. org/10.20982/tqmp.09.2.p079 